\title{
Análisis de la crítica especializada de cine y videojuegos desde la sociología cultural
}

\section{Analysis of the specialized criticism of cinema and video games from cultural sociology}

\author{
Enrique Morales-Corral \\ Universidad de Valladolid \\ emorales@uva.es \\ Javier Gayo-Santacecilia \\ U-TAD \\ javier.gayo@u-tad.com
}

\begin{abstract}
Resumen: Este artículo pretende mostrar, a partir de un análisis comparativo desde la sociología cultural del efecto proyectivo tanto en el consumo de videojuegos como en el de cine a partir de la valoración de la crítica realizada en revistas especializadas de ambos sectores ya sea en papel u online. Para ello se profundiza en el análisis cuantitativo de los veinte productos con mayores ingresos de estas dos industrias culturales durante el pasado 2016, comparando elementos como los códigos de edad recomendados para ambos productos o géneros específicos.

Entre los principales resultados podemos observar cómo hay una mayor o mejor valoración por parte de la crítica especializada acerca los videojuegos más vendidos, respecto a las películas con mayor recaudación de taquilla, y cómo hay diferencias significativas en las recomendaciones de edad para estos productos.

En la industria cinematográfica solo cuatro películas españolas se han situado entre las veinte primeras, mientras que ningún videojuego español ha conseguido situarse entre los más vendidos. También es importante destacar el gran número de películas con una categoría apta para niños en esta clasificación, alcanzando dos de cada tres películas aptas para personas menores de doce años, ofreciendo así un panorama de más cine familiar frente a los videojuegos, más diversos respecto a este factor.
\end{abstract}

Palabras clave: industrias culturales, estudios de videojuegos, estudios de cine, crítica, comunicación. 


\begin{abstract}
This communication aims to show, based on a comparative analysis since cultural sociology, the projective effect of the consumption of video game and film products based on the evaluation given in specialized magazines. It also delves into an analysis of both cultural industries from the 20 products with the highest revenue in 2016, comparing elements such as recommended age codes for both products or specific genres.

Among the main results we can observe how there is a greater evaluation by the critics specialized in the most sold videogames with respect to the films with the highest box-office takings, and how there is significant differences about age rating in both cultural industries.

In the film industry, only four Spanish films have made it into the top 20, unfortunately there isn't any Spanish videogame. It is also important to highlight the large number of films with a category suitable for children in this classification, reaching two out of three films suitable for children under 12 years of age, thus offering a panorama of more family cinema, videogames are more diverse in this subject.
\end{abstract}

Keywords: cultural industries, game studies, cinema studies, critical, communication. 


\section{INTRODUCCIÓN}

La importancia de las industrias culturales en la sociedad actual sigue creciendo año tras año. Junto con los nuevos gadgets o nuevos elementos electrónicos (consolas de videojuegos, e-books, smartphones, tablets...), las industrias culturales están formando una simbiosis perfecta, ya que se alimentan entre sí formando un elemento comercial y social de primer orden. Los gadgets son el hardware, son las máquinas que nos entretienen, mientras que las industrias culturales son el software, el contenido, lo que realmente colma nuestros sentidos y nos hacen disfrutar, emocionarnos, sufrir..., en definitiva, experimentar sensaciones; siempre hay algo para todos los gustos y bolsillos.

Cuando hablamos de industrias culturales, no podemos dejar de mencionar la Escuela de Frankfurt, cuya principal contribución en este campo fue mostrar la expansión de este mercado como una forma específica de extensión cultural, con un nuevo tipo de producción: la cultura de masas y la aplicación de los principios de la organización del trabajo taylorista al desarrollo cultural. Se trata de una nueva forma de entender la cultura como un nuevo sector económico por derecho propio y con un mercado muy grande, alimentado con las constantes necesidades de la nueva clase media, deseosa de un flujo constante de cultura (Horkheimer y Adorno, 1988: 1). Su necesidad cultural es insaciable gracias a la accesibilidad que el Estado permitió a la educación media y superior de toda la población de los países industrializados (Levy, 2007). Se habla incluso de un proceso de democratización de la cultura que permite una amplia difusión de los contenidos al público en general, los hijos del proletariado, en forma de acceso a la educación, que los padres legan a sus hijos al permitir un crecimiento económico estable y constante.

De esta manera, lo que empezó siendo una adquisición social-cultural se convierte hoy de facto, mediante las comunicaciones y los algoritmos de búsqueda, en un «derecho» que se arraiga y defiende a ultranza desde el lado del consumidor, estando íntimamente ligado al consumo fagocitado y retroalimentado de las opiniones propias, o en muchas ocasiones basadas en información sesgada o limitada de los medios.

«Los consumidores han aprendido a recibir exactamente lo que quieren (básicamente vía Internet), lo que está afectando también a su comportamiento en otras situaciones de consumo, incluso en el ámbito no-digital» (Arbaiza, 2013: 192). Así encontramos medios que buscan complacer antes que informar, posicionar más que valorar, derivando en la adquisición de nuevas rutinas comunicativas entre la población general.

En términos de Horkheimer (1998: 29), «una industria cultural de gran producción conspicua, en la que la exposición toma conciencia de sí misma, siendo parte inherente de la esencia». Este proceso está estrechamente vinculado al progreso tecnológico, especialmente en el ámbito de la tecnología de la información. Actualmente, la necesidad 
de conexión online para casi todo es un nuevo proceso que por acumulación afecta a todo el sistema. Dentro de esta conectividad ubicua y permanente, la velocidad de búsqueda y procesado de información también permite más fácilmente sesgar la información, como planteaba Watson: «Ya estamos tan conectados a través de las redes digitales que se ha desarrollado una cultura de la respuesta rápida. En la actualidad estamos siempre tan disponibles que no nos damos tiempo para pensar de forma adecuada sobre lo que estamos haciendo» (Watson, 2011: 12).

Son productos diseñados para ser consumidos rápida y constantemente, que necesitan nuestra atención continua, o podemos pasarnos algo importante por alto. El proceso parece una telaraña en la que cada día te involucras más y más, captando tu atención de manera constante.

Al mismo tiempo, en este juego de producción-consumo, el consumidor se encuentra siempre en tierra de nadie, entre la necesidad de aceptación e integración dentro de la tendencia y la búsqueda de la diferenciación en su asumido papel solipsista, como nos recordaba Martucelli: «Si los individuos siguen siendo socializados a través de factores culturales, la socialización opera en un contexto social en el cual la cultura posee cada vez más un rol ambivalente. Ella ya no es más solamente la garante del acuerdo durable entre el actor y la sociedad (como lo fue en mucho en las sociedades culturalmente cerradas o en la tesis del personaje social), sino que aparece como un agente permanente de diferenciación» (Martucelli, 2010: 16).

El videojuego encaja perfectamente en este modelo, siendo hoy en día uno de sus máximos exponentes. Según datos de la consultora especializada NewZoo, ya somos más de 2.200 millones de jugadores en todo el mundo y en aumento, siendo China el país con mayor número y el smartphone el dispositivo más común para jugar, lo que representa un negocio de más de 94.500 millones de euros (NewZoo en DEV, 2017).

Según Zallo (1992), el estado de la investigación económica en las industrias culturales es mínimo, debido a su falta de sabiduría o ciencia y a su sesgo cuando no existen, siendo más marcado en el caso de la investigación española. Esta situación no ha cambiado significativamente desde entonces, no solo por causas de falta de financiación, sino de tradición y voluntad política en referencia a estos asuntos. Entre las razones que presenta este autor para la falta de investigación y que no han cambiado significativamente se encuentran las siguientes:

- El ocultismo de la información corporativa. Demostrando una falta de transparencia entre las empresas privadas de los sectores referenciados.

- Pereza institucional. Propiciando que estudios relacionados sobre el tema se realicen con un apoyo público mínimo. 
- La insuficiente tradición de investigación universitaria en los estudios macroeconómicos y sectoriales de comunicación. No existe un corpus investigador sólido que afronte esta problemática en España.

En la tradición neoclásica de la economía, todo el sector de las TIC (tecnologías de la información y la comunicación) se está considerando ahora como parte de un conjunto más amplio de un nuevo sector económico. La función social, sus formas particulares de trabajo, el contenido creativo y las prácticas sociales deben ser considerados. Todo esto nos lleva a pensar en la producción de la comunicación como un área productiva específica en la que la incidencia del cambio técnico es fundamental.

Como dice Zallo (1992: 37), «lo productivo no tiene nada que ver con la forma del producto final, sino con la naturaleza del trabajo y el proceso de valoración que tiene lugar previamente». Esta visión del autor hace que todos los sectores que intervienen en la elaboración de productos culturales se sientan realmente partícipes del proceso, formando una verdadera estructura laboral y económica digna de ser estudiada minuciosamente como un sector económico propio. Es decir, se está produciendo un proceso de creciente simbolización de todas las producciones para el consumo. El gasto en bienes culturales se ideologiza otorgándole un valor, no solo material - la forma en que han sido adquiridos-, sino también cultural y de prestigio social, ya que la concepción de lo cultural en nuestra sociedad trasciende el sentido original de la alimentación del alma, y este estímulo es suficiente para que los «estudiosos» continúen sus respectivas investigaciones.

En la sociedad de la que formamos parte, lo cultural trasciende lo material y se convierte más en una ostentación que en un alimento para el espíritu. No solo debemos saberlo todo, sino también promulgarlo, buscando el estatus, la identidad, el buen gusto...

Desde esta perspectiva y conectando con el pensamiento de Baudrillard (2009: XXXI) los consumos culturales quedarían encerrados en una «actividad sistémica de uso expresivo e identificativo de signos», es decir, que dichos consumos culturales se convierten a su vez en un significante social, trascendiendo el complejo sistema recreado por los actores mediáticos.

En los elementos culturales, cuando hay corporeidad, es simplemente un vehículo, un soporte que el mercado necesita para reproducirse de forma magistral y convertir los elementos culturales en industrias culturales, promoviendo su realidad como elemento económico de primer orden (Bustamante, 2003). Se produce un proceso de elevación del propio «vehículo», convirtiendo al propio medio como elemento heterogéneo de un proceso que trata de revertir sobre todo el proceso, dejando en muchos casos la obra fuera o sin un lugar ejemplar. El medio en muchos casos sustituye al propio mensaje, 
desarrollando una de las premisas más significativas de los estudios realizados por McLuhan (1974).

Dentro de las industrias culturales, una en particular siempre ha atraído nuestra atención, ya que hemos estado rodeados de su influencia desde nuestra tierna infancia: los videojuegos. Esta industria cultural, tan boyante hoy en día, no siempre ha gozado de la aprobación del mundo académico, la crítica y el público. Los videojuegos son una industria cultural plural que mezcla géneros creando los suyos propios, absorbiendo parte de la esencia de los demás y añadiendo el elemento fundamental de su éxito, al menos para nosotros: la interacción, un elemento que contrasta con la pasividad del usuario/espectador de las otras industrias culturales. El videojuego arrastra la necesidad de ser parte de la historia y entrar en ella de una manera más profunda que el resto, haciendo al usuario espectador activo e introduciéndose en la trama o historia al practicar las mecánicas del juego planificadas por los creadores.

Al revisar la bibliografía, nos ha sorprendido la falta de estudios comparativos y, más específicamente, la escasez de todo lo que tiene que ver con la crítica de videojuegos, y es que esta floreciente industria carece de estudios rigurosos y comprometidos que puedan formar una ciencia en torno a su desarrollo (Morales, 2015). Es necesario que todas las disciplinas integren esta industria cultural como elemento básico en los mecanismos de comunicación, ya que su influencia sigue creciendo año tras año. La universidad como creadora de ciencia debe abordar este tema pendiente y mostrar la compleja realidad que tenemos por delante los investigadores.

Este artículo pretende ser un primer paso en este largo y sinuoso camino, mostrando una serie de datos y conclusiones que parten de los veinte videojuegos más vendidos en España en 2016 desde todas las plataformas de consola, basados en cifras de la consultora especializada en esta industria, NewZoo (DEV, 2017), comparando datos con las veinte películas de mayor éxito en España durante 2016, al menos en la taquilla de cine, según datos proporcionados por el Ministerio de Cultura de España (2017) en la sección audiovisual de su web.

Una vez realizado este estudio inicial, el objetivo es continuar recopilando información cuantitativa, a partir de la actualización de los datos de consumo de estas dos industrias culturales, e introducir una perspectiva cualitativa a partir del contraste con expertos de crítica en ambos sectores que puedan ofrecer los matices propios de cada sector cultural a modo de observatorio continuado.

\section{METODOLOGÍA}

Queremos utilizar la Teoría de la Agenda Setting (McCombs, 1985) para explicar el tipo de comportamiento de las principales revistas de la industria de los videojuegos y 
el cine. Por agenda setting entendemos la teoría en la que los medios de comunicación desarrollan una gran influencia en el público, ya que determinan lo que es noticia y lo que no lo es, provocando centros de discusión interesados (Piñuel y Lozano, 2006). Los medios de comunicación pueden valorar la importancia de una noticia u otra en función del tiempo que dediquen a ella y del lugar en que la coloquen en su lista de reproducción. Ellos deciden lo que está en la agenda, lo que es noticia y lo que está excluido (Moragas, 1986), a partir de sus propios intereses o de efectos externos no estructurados, como pueden ser las modas o las circunstancias no inicialmente previstas.

Queremos comparar estas dos grandes industrias culturales para ver si la crítica de ambas reflejaba o no el éxito comercial de un producto. Comprobar cómo los críticos tenían razón sobre lo que el público piensa de los veinte productos de mayor éxito comercial en España durante el año 2016.

En este sentido observaremos también la posible «construcción de realidades» desde la crítica, como un sistema que, autoconstruido, puede decantar un sesgo, ya que en ocasiones se podría llegar a ver que se entrecruzan factores informativos y factores promocionales o incluso de marketing, decantando una visión de manera intencionada o no, pues, como nos recordaba Aladro, «[...] el concepto de información quedó asociado al poder de la determinación y concreción de estados, sucesos o cosas que efectivamente surgen en la realidad. Cuando en las ciencias sociales se hace un uso metafórico o figurado de esta idea, y se afirma que la información y la comunicación en su sentido más extenso crean y construyen realidades, se está indicando esta misma verdad» (Aladro, 2009: 18).

Nuestra intención es comparar el consumo de videojuegos y críticos especializados con el consumo de cine y críticos especializados y ver si existe una relación (Gaitán y Piñuel, 1998). Nuestra principal hipótesis se basa en el hecho de que, a diferencia del cine, donde la crítica tiende a estar en desacuerdo con la taquilla, en los videojuegos, el éxito de ventas de un producto suele ir acompañado de buenas críticas y ahondar en el porqué. Sería necesario un estudio más amplio y profundo para afirmar que el éxito de un videojuego depende en gran medida de buenas críticas, y para comprobar si los compradores de videojuegos consideran o no la evaluación especializada a la hora de comprar este tipo de producto.

Para los datos sobre cine, nos hemos basado en los proporcionados por el Ministerio de Cultura español en su página web (https://goo.gl/Krjr6q), que están disponibles gratuitamente desde hace muy poco, los de 2016.

En el caso de los videojuegos, los datos de los veinte juegos de consolas más vendidos en España en 2016 han sido proporcionados por la consultora especializada NewZoo, que suele suministrar los datos a organizaciones como DEV (Asociación Española 
de Desarrollo de Videojuegos), http://www.dev.org.es/, una de las principales impulsoras de la difusión de esta industria cultural en España.

Hemos realizado un protocolo de análisis bajo el programa Excel en el que hemos recogido las reseñas especializadas de ocho revistas de cine y ocho videojuegos con el fin de encontrar una media crítica con la que ver una trayectoria de ambas industrias culturales y así comparar las tendencias de los productos más exitosos desde el punto de vista comercial. El criterio de selección de los medios especializados se ha basado en la disponibilidad online y en papel de las revistas, y en la importancia de trazabilidad en la web a partir del EGM, proporcionado por la Asociación para la Investigación de Medios de Comunicación (AIMC), intentando hacer referencia a los medios más demandados y leídos en ambas prensas especializadas.

Los medios especializados consultados en videojuegos y cine son los siguientes:

TABLA 1

\begin{tabular}{ll}
\hline \multicolumn{1}{c}{ Revistas de Videojuegos } & \multicolumn{1}{c}{ Revistas de Cine } \\
\hline 1 Meristation - online & 9 Fotogramas - papel y online \\
\hline 2 3D Juegos - online & 10 Cinemanía - papel y online \\
\hline 3 Metacritic - online & 11 Filasiete.com - online \\
\hline 4 Hobbyconsolas - papel y online & 12 Elséptimoarte.com - online \\
\hline 5 Vandal.net - online & 13 Hobbyconsolas - online \\
\hline 6 IGN-Spain - papel & 14 Filmaffinity - online \\
\hline 7 Mundogamers - online & 15 IMDB- online \\
\hline 8 ÁreaJugones-online & 16 Metacritic - online \\
\hline
\end{tabular}

Fuente: elaboración propia.

\section{RESULTADOS}

Para ambas industrias culturales nos hemos basado en análisis generales de productos sin reflejar análisis parciales de elementos específicos. En las revisiones de los videojuegos es normal que haya subapartados, destacando secciones específicas, como gráficos, jugabilidad, música...; no los hemos tenido en cuenta y hemos optado por recoger únicamente las notas finales de los productos analizados para poder comparar productos completos. En la crítica de cine esta diferenciación no es habitual, y es nor- 
mal que solo haya una nota final para dar al lector una valoración y ayudarle en su opinión. Todas las notas se han convertido fielmente a la conversión de 0 a 100 para facilitar su visualización y análisis.

En el Gráfico 1 podemos ver la trayectoria de las notas críticas finales de ambas industrias culturales en los veinte productos más exitosos en España durante el 2016. A simple vista se observa cómo hay una mayor aceptación de la crítica especializada en productos de videojuegos: podemos ver claramente cómo la línea de los medios de crítica de videojuegos (la roja) es superior a la de la crítica de cine (la azul), por lo que se cumple la hipótesis principal inicial.

Sería necesario ver en investigaciones posteriores si se trata de una tendencia que se repite año tras año o incluso aumentar el número de productos a analizar. También sería interesante ver el desempeño real de los productos comparando la relación coste-beneficio en ambos sectores culturales o incluso analizar el costo cultural en diferentes sectores. Las diferencias además son notables y no hay duda sobre la conjunción entre crítica y ventas en el mundo del videojuego, quedado mucho más relegado el proceso de crítica y su éxito comercial en el mundo del cine.

Se trata, por lo tanto, de un buen punto de partida para un experimento mayor que ahonde en estos temas, intentando profundizar en algunos de los aspectos ya señalados e incorporando nuevos, que nos permitan ofrecer una visión más específica de esta interesante relación entre crítica y ventas, entre estas dos grandes y relevantes industrias culturales. Uno de los objetivos que podríamos marcarnos a la hora de analizar esta relación

\section{GRÁFICO 1}

Comparación de los videojuegos y las medias de las películas

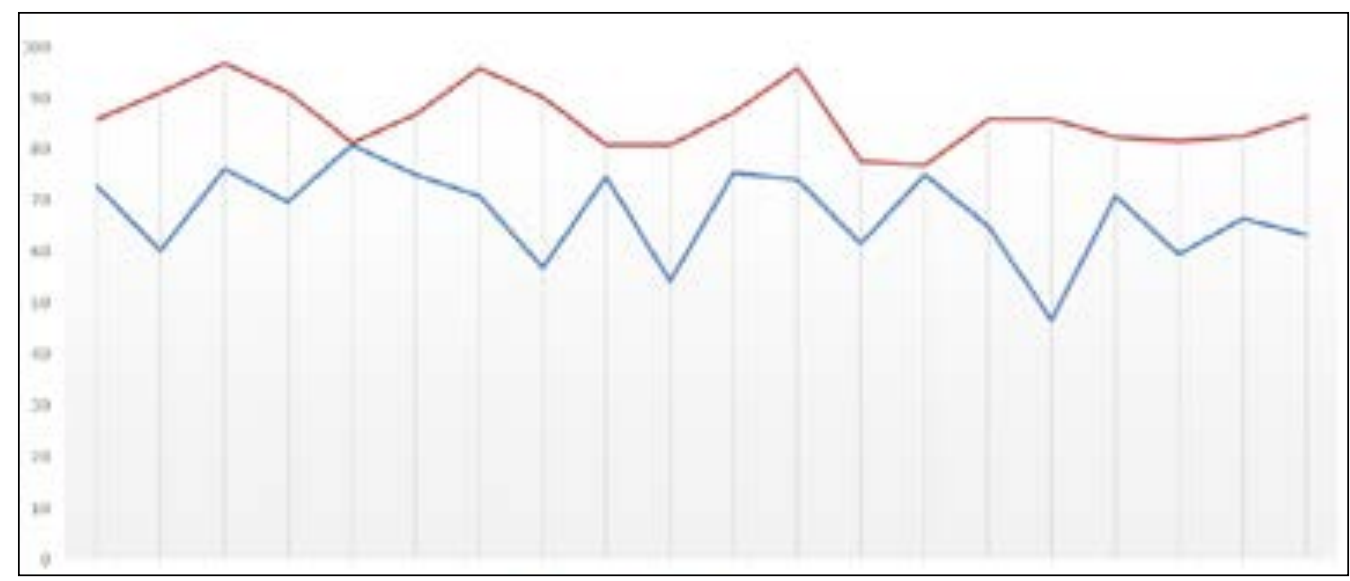

Fuente: elaboración propia. 
sería fijarnos también en el proceso de cómo se realizan las críticas, quién las hace y con qué fin: informar, exponer criterios, analizar... Este matiz ayudaría sin duda a obtener una visión más integral de los datos enmarcándolos dentro de una teoría cultural propia.

También podemos observar en estos dos gráficos (2 y 3 ) que hay una vida comercial más alargada en los videojuegos, ya que hay seis juegos que son anteriores al año de análisis de los datos de ventas; uno de ellos incluso fue publicado en 2014, dos años antes de la fecha analizada, 2016. Este es un ejemplo de la larga vida comercial que los videojuegos pueden tener en el mercado a pesar de un mundo comercial cada vez más competitivo y obsesionado con los avances tecnológicos (Morales, 2011); hay productos que triunfan basados en la parte creativa y en su efecto lúdico, a priori los puntos principales de un buen videojuego. En este caso particular el videojuego es GTA V, que ha tenido un componente online que ha extendido la vida comercial del juego, incluso hasta hoy en día. Esto contrasta con los datos recogidos en este estudio sobre las películas analizadas; solo hemos tenido en cuenta los ingresos de taquilla. Somos conscientes de que el análisis debe ampliarse, ya que una película también tiene una vida comercial cuando se edita en formato físico, ya sea DVD o Blu-ray. Estos datos no se recogen y quizás sería interesante realizar un estudio que los incluyera, así como los beneficios del formato VOD (Video on Demand), tan de moda últimamente por la aparición de nuevos actores, como Netflix, HBO..., de los productos cinematográficos para hacer una mejor comparación con los videojuegos.

En cuanto a los estrenos de películas, la proporción es del $85 \%$ en 2016 frente a solo el $15 \%$ en 2015 de las veinte películas más vistas en el cine durante 2016. Estos datos son más lógicos porque pocas películas pueden resistir en los cines durante más de unas semanas; solo fenómenos como Star Wars pueden tener muchas semanas su película en la cartelera; las otras dos películas son españolas y se estrenaron en los últimos días del 2015.

\section{GRÁFICOS 2 Y 3}

Fecha de lanzamiento de videojuegos y películas
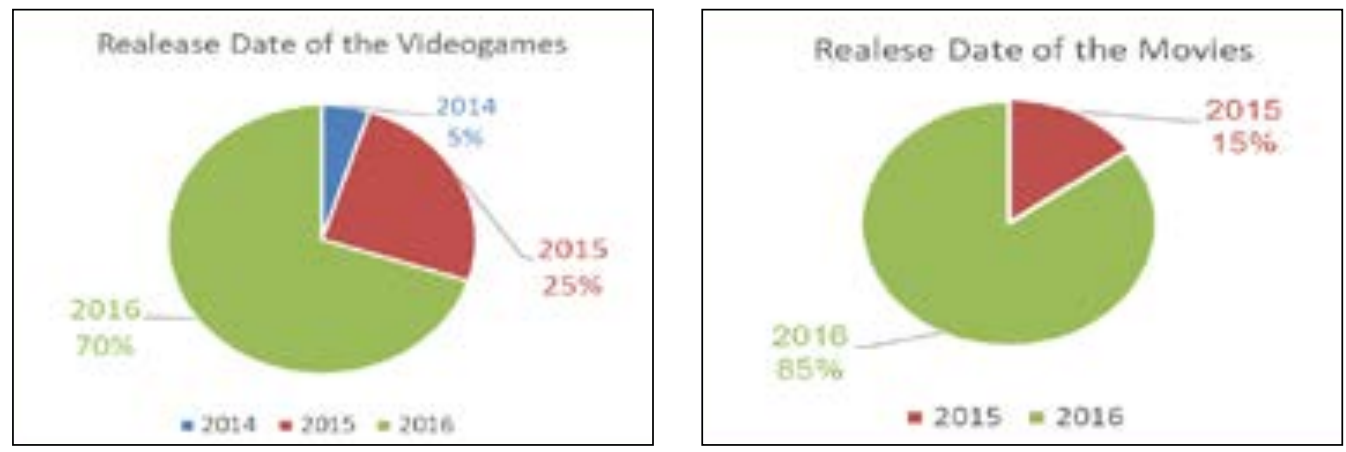

Fuente: elaboración propia. 
Otro punto importante a la hora de analizar y comparar estas dos industrias culturales es la nacionalidad de la producción (Gráficos 4 y 5). En cine, hay una clara ventaja para las producciones estadounidenses, que cubren el $75 \%$ de las películas de mayor taquilla, con una participación española de solo el $20 \%$, es decir, solo cuatro películas españolas han conseguido estar entre las veinte primeras de taquilla en 2016.

\section{GRÁFICOS 4 Y 5}

Cine Nacionalidad y Videojuegos Nacionalidad
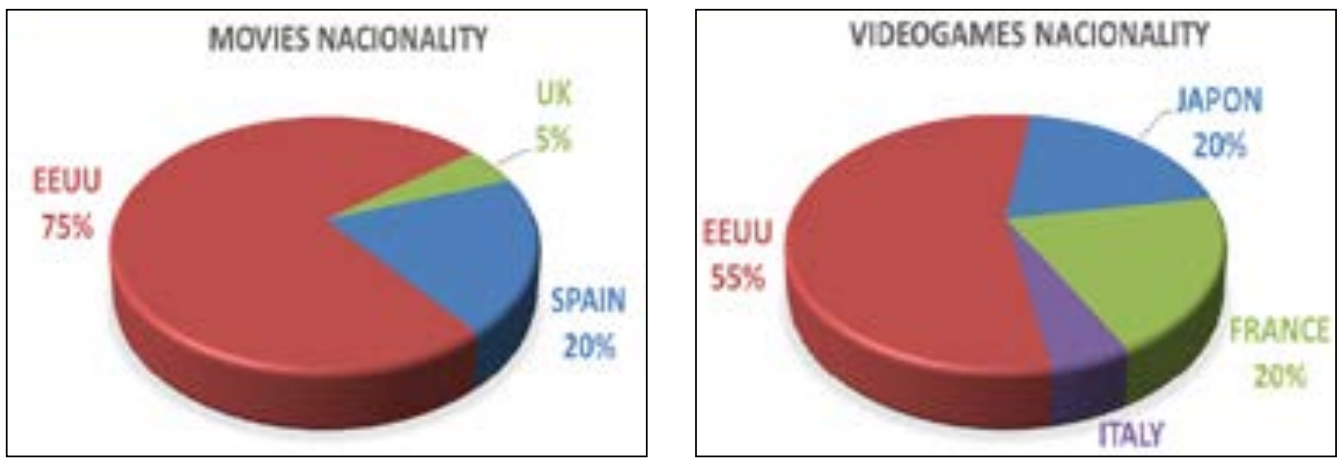

Fuente: elaboración propia.

En cuanto a los videojuegos, el país de producción por excelencia es EE. UU., con el $55 \%$ de las producciones. Japón tiene que conformarse con el $20 \%$ de las producciones y España no tiene representación, algo triste y preocupante para un mercado que alcanza los 1.557 millones de euros en 2016.

Otro aspecto para destacar es la clasificación por edades asignadas a las películas y los videojuegos. En el mundo de los videojuegos existe un acuerdo europeo, el código PEGI (Pan European Game Information, http://www.pegi.info/es), que, además de una clasificación por edades (Figura 1), incorpora una serie de pictogramas (Figura 2) en los que es posible observar contenidos que no son apropiados para un público infantil o juvenil, mostrando a padres y educadores que, por ejemplo, hay insultos o referencias explícitas a las drogas o al sexo en el juego.

Es el primer código del sector del entretenimiento que cumple con los diferentes estándares culturales de los veinte países europeos que lo han suscrito. El código entró en vigor en España en junio de 2003.

Su principal objetivo es garantizar que los consumidores, y en particular los padres y educadores, dispongan de información suficiente sobre los videojuegos que se comercializan en España o en cualquier otro país europeo perteneciente al sistema para poder 
elegir los productos que mejor se adapten a la edad del usuario, limitando así la exposición de los niños a contenidos que puedan ser inadecuados para ellos. La adopción del nuevo código amplía y sustituye al sistema de autorregulación creado por aDeSe (actualmente DEV) para España, que entró en vigor el 1 de abril de 2001 y al que se había adherido la práctica totalidad del sector.

\section{FIGURA 1}

Código recomendado de mediana edad para los videojuegos.
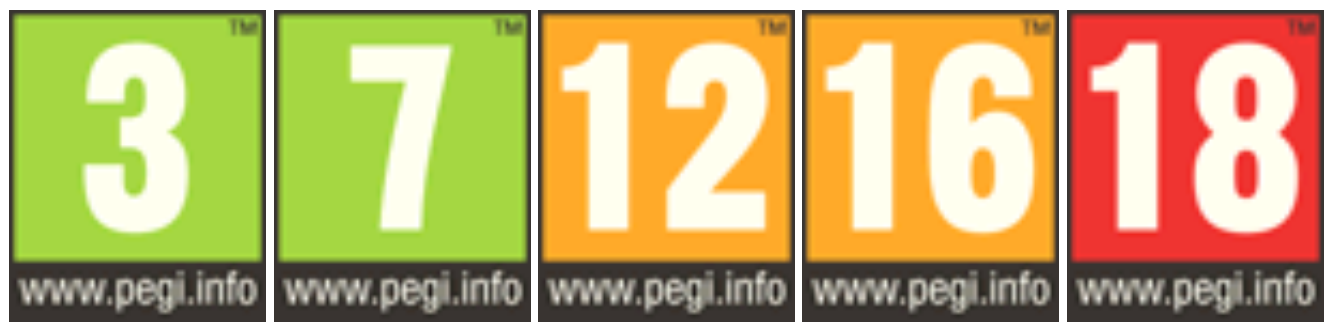

Fuente: Pegi (https://pegi.info/)

Iconos descriptivos de contenido o pictogramas que especifican por qué el juego ha sido clasificado en la categoría de edad seleccionada. Los iconos utilizados son los siguientes:

\section{FIGURA 2}

Iconos descriptivos del contenido

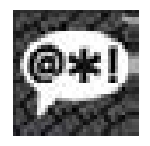

Mal lenguaje. El juego contiene insultos o palabras malsonantes.

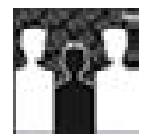

Discriminación. El juego contiene representaciones discriminatorias.

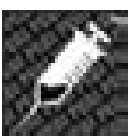

Drogas. El juego hace referencia o muestra el uso de drogas.

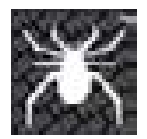

Miedo. Jugar a este videojuego

puede asustar.
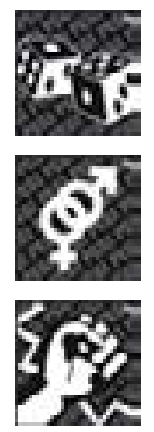

Apuestas. Juegos que fomentan el juego y las apuestas o que enseñan a apostar.

Sexo. El juego contiene comportamientos sexuales o referencias sexuales.

Violencia. El juego contiene representaciones violentas.

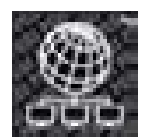

En línea. El juego se puede jugar en línea.

Fuente: Pegi (https://pegi.info/). 
En cuanto a los códigos de edad para las películas, el Ministerio de Cultura de España presenta la siguiente clasificación:

- TP: Todos los públicos admitidos.

- TP-E: Todos los públicos admitidos y recomendados para los niños.

- NR-7: No recomendado para personas menores de 7 años.

- NR-12: No recomendado para personas menores de 12 años.

- NR-16: No se recomienda para personas menores de 16 años.

- NR-18: No se recomienda para personas menores de 18 años.

- X: Pornografía, defensa de la violencia

Si analizamos nuestros registros (Gráfico 6), podemos ver cómo hay una gran dispersión dentro del código de edad entre las veinte películas más vistas de 2016 . Y en el Gráfico 7, la distribución del PEGI en los videojuegos.

\section{GRÁFICOS 6 Y 7}

Edad recomendada en películas y videojuegos
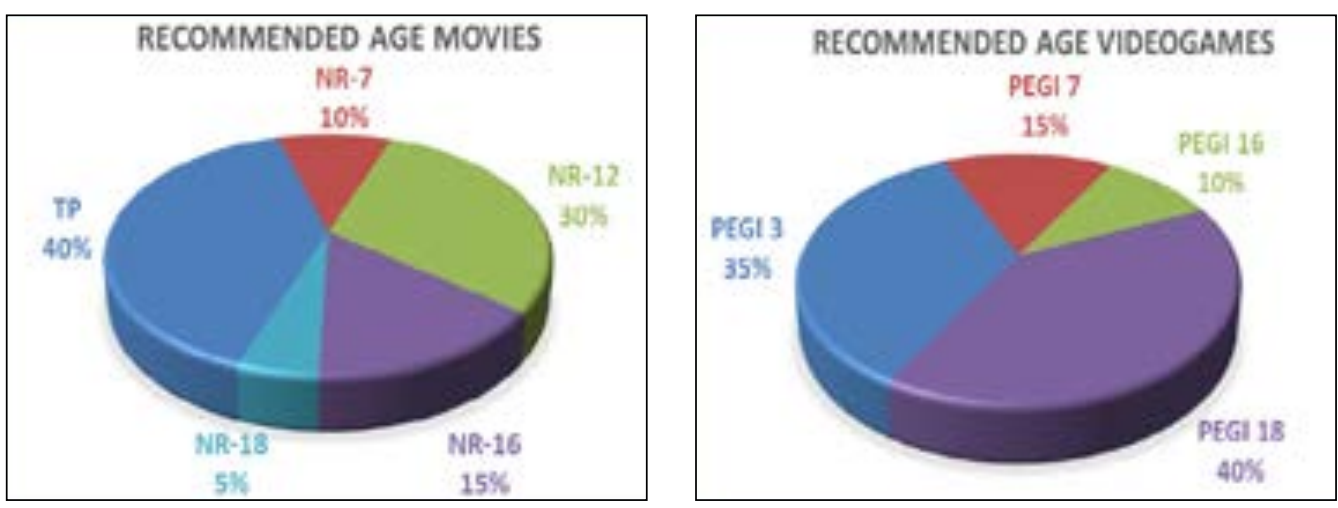

Fuente: elaboración propia.

A partir de estos datos, podemos ver que la mitad de las películas están especialmente dirigidas a los niños, con un porcentaje para todos los públicos del $40 \%$ y de las no recomendadas para personas menores de 7 años, con un $10 \%$. Esto demuestra claramente cómo la taquilla española de 2016 ha reconocido este tipo de productos como los más demandados por el público. Las películas para adultos representan solo el $5 \%$ del total, siendo un tipo de película poco rentable en taquilla; habría que ver si en otros formatos de exhibición la situación cambia, dejando este aspecto para siguientes análisis. El público no exige productos de calidad para adultos, se entretiene con productos de carácter más familiar, mostrando al cine su faceta más globalizadora. 
En cuanto a los videojuegos, se alcanza el mismo porcentaje: la mitad de todos los videojuegos son aptos para niños mayores de 3 años. Este caso es posiblemente un espejismo porque estos juegos son juegos de deportes, en particular versiones del juego FIFA (juegos de fútbol), que no tienen esta edad de control. Destacan los juegos recomendados a partir de los 18 años con un $40 \%$ del total, lo que asegura un mercado importante para este tipo de juegos, especialmente los juegos de disparos, como la saga Call of Duty o los títulos de Battlefield, un contraste con los datos ofrecidos por las películas y que abre un debate interesante sobre si realmente estos videojuegos están siendo jugados por personas de más de 18 años o no.

\section{CONCLUSIONES}

La principal conclusión es que la hipótesis que nos planteamos al principio del artículo se confirma. Se observa que los veinte videojuegos más exitosos de España durante 2016 presentan una puntuación media crítica mayor a la puntuación media crítica de las veinte películas más exitosas durante el mismo periodo (Gráfico 1). Esto confirma que las críticas de videojuegos están más en sintonía con los gustos de los usuarios que las críticas del cine con sus espectadores. Parece que los motivos del público para ir al cine a ver una película u otra no se basan especialmente en la crítica especializada, sino en otros factores. Está por demostrar si sí existe referencia o influencia directa en la compra de videojuegos a partir de las críticas de estos, pero queda pendiente dicho análisis para futuros estudios.

De hecho, este es un estudio exploratorio que nos ha permitido fijar una serie de nuevos objetivos con los que seguir estudiando y analizando esta importante premisa en dos de las más exitosas industrias culturales del momento. Se trata de ahondar en cómo se realizan las críticas especializadas, por quién se realizan, con qué fin y qué utilidad tienen, y si tienen o no relevancia en los usuarios/espectadores a la hora de elegir uno u otro producto cultural. Para ello emplazamos al lector a posteriores estudios. En esta línea ha sido clave la simbolización que estas industrias han llevado a cabo en las últimas décadas, favoreciendo la especialización de la narrativa y la conectividad ubicua y permanente.

También son interesantes los análisis de los códigos de edad de los productos analizados, y podemos observar cómo en ambas industrias culturales hay abundancia de contenidos aptos para personas menores de siete años. En el análisis de las nacionalidades de producción, EE. UU. destaca en ambas industrias culturales, siendo el papel de 
España muy limitado en el cine y casi inexistente en los videojuegos de mainstream, una tendencia preocupante que debería abordarse en los próximos años.

\section{BLIBLIOGRAFÍA}

Aladro Vico, E. (2009). La Información Determinante. Editorial Tecnos. Grupo Anaya, S.A.

Arbaiza Rodríguez, F. (2013). «Orígenes de la customización masiva: Las interacciones individuales consumidor-marca dentro de mercados masivos en el marketing». Revista de Comunicación, 12. Dialnet.unirioja.es

Baudrillard, J. (2009). La sociedad de consumo. Sus mitos, sus estructuras. Siglo XXI de España Editores, S.A. Madrid.

Bustamante, E (coord.) (2003). Hacia un nuevo sistema mundial de comunicación. Ed. Gedisa.

DEV (2017). Anuario 2017 del Desarrollo Español de Videojuegos. [https://goo.gl/UorsRv].

Gaitan Moya, J.A. \& Piñuel Raigada, J.L. (1998). Técnicas de investigación en comunicación social. Ed. Síntesis.

Horkheimer, M. \& Adorno, T. (1988). Dialéctica del ilumnismo. Ed. Sudamericana. Buenos Aires.

Horkheimer, M. (1998). Dialéctica del a ilustración: Fragmentos Filosóficos. Ed. Trotta. Madrid.

Levis, D. (1997). Los videojuegos, un fenómeno de masas. Ed. Paidós de Comunicación.

Levy, P. (2007). Cibercultura. la sociedad de la sociedad digital. Ed. Anthropos.

Martuccelli, D. (2010). «La individuación como macrosociología de la sociedad singularista». Persona y Sociedad / Universidad Alberto Hurtado. Vol. XXIV, n. ${ }^{\circ}$ 3, 2010, 9-29

McCombs, M. (1985), «La comunicación de masas en las campañas políticas: información, gratificación y persuasión», en Moragas, M. de (eds). Sociología de la Comunicación de Masas. Estructura, funciones y efectos. Ed. Gustavo Gili, S.A., Barcelona.

McLuhan, M. (1974). La cultura es nuestro negocio. Ed. Diana. México.

Ministerio de Cultura de España (2017), Informe anual sobre el cine en España. [https:// goo.g1/vahHX2]

Moragas, M. (1986). Sociología de la comunicación de masas. Estructura, funciones y efectos. Ed. Gustavo Gili. Barcelona. 
Morales, E. (2011). «El videojuego y las nuevas tendencias que presentan al mercado de la comunicación». Anuario electrónico de estudios en Comunicación Social Disertaciones. Vol. 4, n. ${ }^{\text {2 }}$, pp. $36-54$ [https://goo.gl/FSXXVC].

Morales, E. (2015). La nueva cultura multimedia en la era digital: el caso del videojuego. Ed. UCM, e-Prints. [https://goo.gl/2VdapW].

PEGI. Pan European Game Information [https://goo.gl/3vp7RB].

Piñuel, J.L. \& Lozano, C. (2006). Ensayo general sobre la comunicación. Ed. Paidós

Watson, Richard. (2011). Mentes del Futuro. ¿Está cambiando la Era Digital nuestras Mentes?. Ed. Viceversa. Ayer y Hoy.

Zallo, R. (1992). El mercado de la cultura. Ed. Gakoa

\section{BREVE FICHA TÉCNICA DEL ESTUDIO}

- Definición de los objetivos de la investigación: recopilación de los datos de las críticas especializadas de películas estrenadas en cine y videojuegos comerciales.

- Descripción del universo: listado de las veinte películas con más taquilla y los veinte videojuegos con más ventas durante 2016 en España.

- Fuente datos de videojuegos: DEV a partir de los datos de la consultora NewZoo (2016).

- Fuente datos de películas: Ministerio de Cultura de España (2016).

- Medios críticos consultados: ver Tabla 1.

- Tratamiento de los datos: realización de las medias aritméticas numéricas adaptadas en la escala de 0 a 100.

- Periodo de recogida de la información: año 2016.

- Visualización de los datos: ver Tabla 1. 